\title{
Variations in carotid sinus anatomy and their relevance to carotid interventions
}

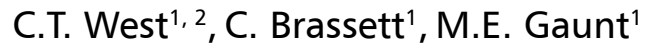 \\ ${ }^{1}$ Human Anatomy Teaching Group, Department of Physiology, Development and Neuroscience; \\ University of Cambridge, United Kingdom \\ 2Digestive Diseases Department, Surrey and Sussex NHS Healthcare Trust, United Kingdom
}

[Received: 23 October 2017; Accepted: 11 February 2018]

\begin{abstract}
Background: The carotid sinus (CS) is a dilatation in the carotid bifurcation usually at the origin of proximal internal carotid artery (ICA). It contains baroreceptors which influence blood pressure. Variations in the location of the CS are of importance as atheromatous plaque commonly forms in this area and procedures such as carotid endarterectomy are performed to reduce the risk of stroke. Inadvertent stimulation of the CS baroreceptors during interventions can have profound effects on the patient's haemodynamic status both intra- and postoperatively, causing serious complications. The aim of this study is to determine the inter-and intra-individual variations in the location of the CS.

Materials and methods: Eighty-two carotid arteries were dissected bilaterally from 41 cadavers. The locations of the CS were noted and divided into four potential sites.

Results: The commonest site is the origin of the ICA (74.3\%), but the CS can also be found in the distal part of the common carotid artery (CCA) inferior to the bifurcation (17.1\%); at the bifurcation involving the distal CCA and origins of both the external carotid artery (ECA) and ICA (7.32\%); and at the origin of the ECA (1.22\%). In individual cadavers, the CS was located at the origin of the ICA in $97.6 \%$ on at least one side. The sites of the CS were asymmetrical in $34.1 \%$. Conclusions: Clinicians performing carotid interventions should be aware of these anatomical variations to avoid inadvertent stimulation of the CS which can cause profound bradycardia and hypotension. (Folia Morphol 2018; 77, 4: 693-697)
\end{abstract}

Key words: carotid sinus, carotid endarterectomy, common carotid artery, internal carotid artery, external carotid artery

\section{INTRODUCTION}

The anatomy of the human carotid arterial system conventionally describes the common carotid artery (CCA) ascending in the neck and bifurcating at the upper border of the thyroid cartilage into the internal carotid artery (ICA) and external carotid artery (ECA). The carotid sinus (CS) is a neurovascular structure visualised as a focal dilatation of the arterial wall typically situated at the origin of the proximal ICA. Anatomical variation in the level and site of the CCA bifurcation and the exact location of the CS within the carotid arteries has significant consequences for the safety and efficacy of clinical interventions performed in this area. The dilatation of the CS has previously been shown to be located in the CCA or ECA as well as the ICA [3]. 


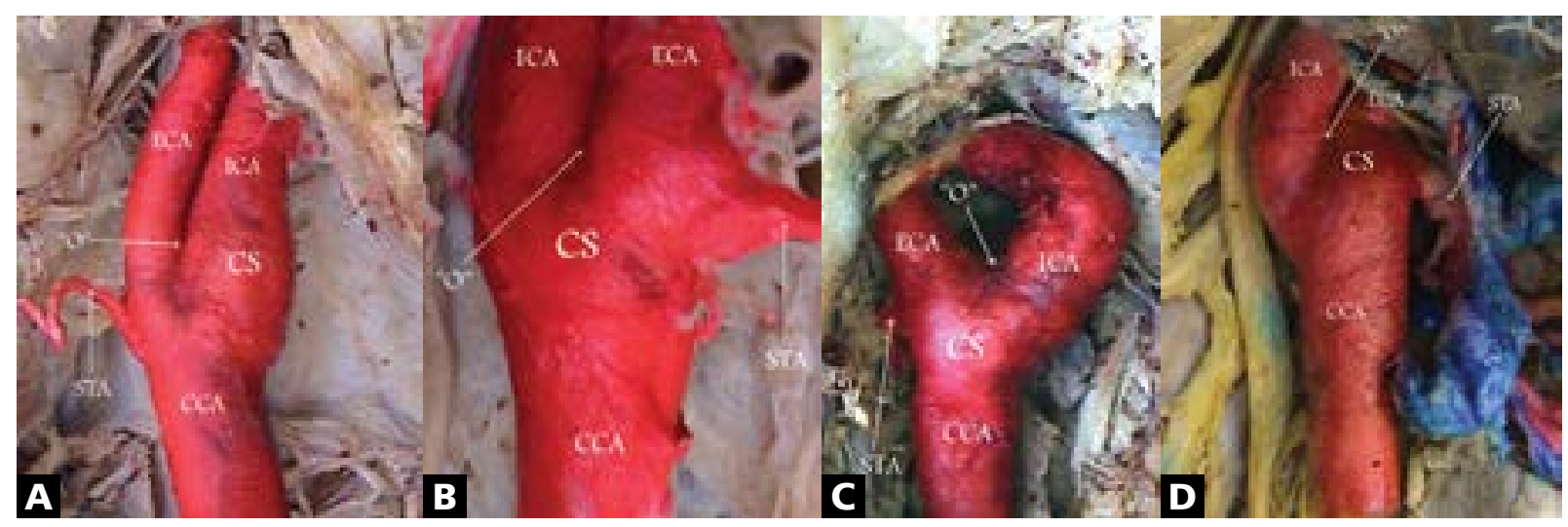

Figure 1. A-D. Dissections of the coronary sinus (CS) in four different positions found. Red tissue denotes arterial tissue, blue venous; STA - superior thyroid artery; 0 - apex of common carotid artery (CCA) bifurcation. A. Site 1: CS is at the origin of the internal carotid artery (ICA) and part of the distal common carotid artery (CCA); B. Site 2: CS is at the distal CCA, including the origin of the ICA and the origin of the external carotid artery (ECA); C. Site 3: CS is at the distal CCA, but caudal to the CCA bifurcation with no extension into the ICA or ECA; D. Site $4:$ CS is at the proximal ECA and part of the distal CCA.

In the wall of the CS are neural elements which are stretch receptors known as baroreceptors, from which afferent fibres run via the carotid sinus and glossopharyngeal nerves to the nucleus tractus solitarius in the brainstem [21]. Efferent impulses are conveyed by the autonomic nervous system to affect the heart rate and vasomotor tone. This is known as the CS baroreflex; typically, a rise in blood pressure stimulates the CS stretch receptors, which triggers a reflex bradycardia and systemic vasomotor dilatation to lower the blood pressure [20].

The carotid bifurcation is a common site for atheromatous plaque formation which, in turn, is a significant cause of thromboembolic stroke [24]. Clinical interventions such as surgical carotid endarterectomy (CEA), carotid angioplasty and stenting significantly reduce the risk of stroke [23]. These treatments, however, are associated with adverse haemodynamic changes due to stimulation of the CS baroreflex during open dissection in CEA or stretching of the CS by an angioplasty balloon or metal stent [4]. Additionally, surgical damage to the CS region can result in marked instability in post-operative blood pressure, particularly hypertension. This in turn may cause serious complications such as haemorrhage, neck hematoma and cerebral hyperperfusion syndrome with resultant intracranial bleeding [17]. There are also documented cases of coronary spasm and resultant myocardial infarction following CS stimulation, but not during CEA or carotid artery stenting $[6,15]$.

Surgeons performing CEA are therefore trained to exercise extra care when dissecting the proximal ICA region, as this minimises stimulation of the CS and reduces these complications. However, if the CS were located in a variant site within the CCA or ECA, inadvertent stimulation is more likely and can produce adverse outcomes. This phenomenon has been observed anecdotally in theatres by the authors. More patients are now undergoing staged bilateral CEA, hence knowledge of whether the site of the CS is consistent in an individual patient would improve safe surgical planning to avoid the above complications. This cadaveric study aims to describe the variations in the location of the CS and determine their inter- and intra-individual variability.

\section{MATERIALS AND METHODS}

A total of 41 embalmed cadavers were used for the study (19 female, 22 male) with mean age at death of 83.5 (range 67-100). Carotid systems were dissected bilaterally, none had had previous carotid surgery so there were no exclusions. Surrounding tissues were removed and the distal $3 \mathrm{~cm}$ of the common carotid, proximal $3 \mathrm{~cm}$ of the internal carotid and proximal $3 \mathrm{~cm}$ were excised in their entirety to allow more detailed examination.

The apex of the bifurcation of the common carotid was used as a common reference point to describe the variations; this is labelled as point " $\mathrm{O}$ " in Figure 1. The CS was defined as the point at which there is a clear dilatation in the lumen from the caudal to the cranial segment of the vessels. The CS was noted to have four discrete locations and was not always isolated to the CCA, ICA or ECA. These locations were designated as sites $1-4$. Comparison of the bilateral sites was also made in each cadaver to determine 


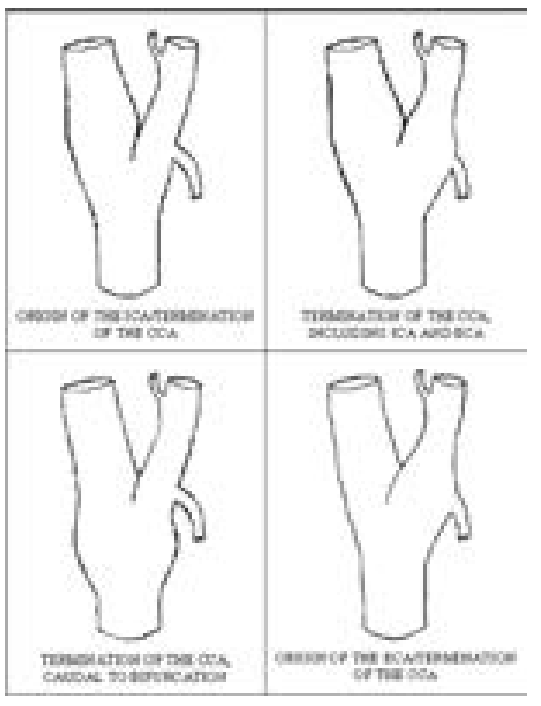

Figure 2. The four different sites of the coronary sinus found in the dissections; abbreviations - see Figure 1.

intra-individual variability. All donors had consented for anatomical research prior to decease in accordance with the Human Tissue Act (2004).

\section{RESULTS}

Four different sites of the CS were identified as shown in Figures 1 and 2. The frequency of sites 1-4 were as follows.

- Site 1: The CS began from the distal part of the CCA and extended into the proximal ICA. This was the commonest site (61 out of 82, 30 left/31 right);

- Site 2: The CS was at the distal CCA and extended into both the proximal ECA and proximal ICA (6 out of 82,4 left/2 right);

- Site 3: The CS was found at the distal CCA, but proximal to its bifurcation with no extension into either the ICA or ECA (14 out of 82, 7 left/7 right);

- Site 4: The CS began in the distal part of the CCA extending into the proximal ECA. This was the least common position (1 out of 82, right side only).

With regards to variation in CS location on the left and right in individual cadavers $97.6 \%$ (37 out of 41 ) had the CS in Site 1 on at least one side. The sites of the CS were asymmetrical in $34.1 \%$ (14 out of 41 ). The sample size in this study was too small to determine any further statistically significant correlation between each side of the neck in individual donors.

\section{DISCUSSION}

The traditional surgical teaching when operating in this area is to take care around the origin of the ICA to avoid stimulating the CS; however, in all four sites the CS also involves the distal CCA. This area should also be handled with the same due diligence. Surgeons should also be aware that in this study $8.54 \%$ of CS also involved the origin of the ECA (site 2 and site 4) and therefore attempts to visualise this area should be made before a traumatic manoeuvre, such as applying a vascular clamp, is performed. It is significant that $34.1 \%$ of CS were found to be asymmetrical in individuals; this implies that prediction of the CS site based on a previous intervention is unreliable.

Carotid artery anatomy is known to be variable even within the same individual [18]. In particular, the vertebral level of the carotid bifurcation and therefore the position of the CS can range from $\mathrm{C} 2$ to the C6-C7 intervertebral disc. This influences where optimal surgical incisions should be made [10]. The branches of the carotid arteries, diameters and degree of tortuosity also vary and have important relevance for clinical interventions $[12,14]$.

The origin of the ICA and terminal CCA is accepted as the most common location of the CS, which is confirmed in the results (74.4\% of carotid systems). However, Binswanger (1879) [2] reported a study of 200 patients that indicated that the CS was present in the ICA in only $40 \%$ of cases. Boyd (1937) [3] had reported the other three less common sites previously, as well as the location of the CS on the ICA distal to the carotid bifurcation which was not noted in this study. Both of these dissection-based papers predate the first reported CEA by Eastcott et al. (1954) [8] and so may not be as relevant for modern interventions; however, they do point out that there has historically been some discrepancy in the location of the CS.

An oversensitive CS baroreflex can result in disabling dizziness, syncope and hypotension. One treatment for this condition is surgical adventitial stripping of the ICA to interrupt the afferent neural pathway and reduce the sensitivity of the baroreflex. Toorop et al. (2013) [22] performed histological examinations on five carotid artery bifurcations. The highest density was found on the medial aspect of the proximal ICA, but some neural tissue was also present in the CCA and ECA. They implied that the CS is only located in the ICA as a guide to performing adventitial stripping for an oversensitive CS. However, the technique is not universally successful and recurrences can occur. To date, additional stripping of the ECA and CCA has not been described but these are two variant locations of the CS. This may explain the failure of this technique 
in some cases. It would be useful to apply modern histological techniques to further compare the pattern and density of neural elements between different areas of the carotid arteries in particular sites where a focal dilatation in keeping with the CS is present.

In an inter-individual and intra-individual study based on the European Carotid Surgery Trial comprising data from 5395 carotid angiograms, considerable differences were found between contralateral carotid bifurcations in terms of vessel diameters, and hence ratios between cross-sectional areas of the CCA, ICA and ECA at the bifurcation [16]. It is thus unsurprising that the location of the CS would also vary, not only between individuals but also between the two sides of the neck in the same individual. In this study, the CS did not have the same location as its contralateral counterpart in $34.1 \%$ bilateral systems. The CS was, however, present in the ICA in at least one side of the neck in $97.6 \%$ of cases.

Stimulation of the CS during procedures such as CEA and carotid artery stenting has been shown to have a range of effects on the cardiovascular status of the patient, both in the peri-operative phase as well as post-operatively [11]. In carotid artery stenting, where there is less tactile control over the stimulation of the CS, atropine has been administrated prophylactically just prior to balloon inflation by the interventionalist or anaesthetist to successfully reduce haemodynamic instability [5].

Atheroma removal during CEA in the region of the CS is associated with a decrease in the sensitivity of CS baroreceptors during and after surgery, specifically type I receptors [17]. The CS baroreceptors are especially important in regulating blood pressure post-operatively; however, these effects do not appear to be long-lasting, with no overall changes on reflex control of blood pressure 6 months after surgery $[7,9]$. The baroreflex function is less likely to be affected following the immediate post-operative period in eversion CEA as opposed to the standard approach using a longitudinal arteriotomy, but the reason for this is not yet clear. Overall the short-term instability following CEA is attributed to the temporary traumatic effects of the surgery [13]. In an effort to mitigate these risks there have been trials to infiltrate the CS nerve during CEA with local anaesthetic in order to attempt to counter the effects of CS stimulation and hence hemodynamic changes peri- and post-operatively. However, most studies have not shown any major benefits $[1,19]$. Reduction in complications therefore depends on good surgical technique, awareness of potential variations in anatomy, close monitoring of the patient intra- and post-operatively and good communication between surgeon and anaesthetist.

\section{CONCLUSIONS}

This study has shown subtle, yet definite, interand intra-individual variations in the location of the $\mathrm{CS}$. The most common location for the CS is at the origin of the ICA, and our results suggest that it is usually in this position in at least one side in the neck. Clinicians performing interventions in this area, in particular CEA, should therefore be mindful of these variations to avoid inadvertent stimulation or allow earlier anticipation so that atropine can be administered prophylactically to mitigate adverse effects from triggering the CS baroreflex.

\section{Acknowledgements}

We acknowledge the kind generosity of our donors and the invaluable assistance of Maria Wright, Darren Broadhurst and Joseph Perfitt in the Human Dissection Room of the University of Cambridge, without whom this study would not have been possible.

\section{REFERENCES}

1. Ajduk M, Tudorić I, Sarlija M, et al. Effect of carotid sinus nerve blockade on hemodynamic stability during carotid endarterectomy under local anesthesia. J Vasc Surg. 2011; 54(2): 386-393, doi: 10.1016/j.jvs.2011.02.036, indexed in Pubmed: 21658884.

2. Binswanger $\mathrm{O}$. Anatomische Untersuchungen über die Ursprungsstelle und den Anfangstheil der Carotis interna. Archiv für Psychiatrie und Nervenkrankheiten. 1879; 9(2): 351-368, doi: 10.1007/bf02666476.

3. Boyd JD. Observations on the human carotid sinus and its nerve supply. Anatomischer Anzeiger. 1937; 84: 386-399.

4. Cao Q, Zhang J, Xu G. Hemodynamic changes and baroreflex sensitivity associated with carotid endarterectomy and carotid artery stenting. Interv Neurol. 2015; 3(1): 13-21, doi: 10.1159/000366231, indexed in Pubmed: 25999987.

5. Cayne NS, Faries PL, Trocciola SM, et al. Carotid angioplasty and stent-induced bradycardia and hypotension: Impact of prophylactic atropine administration and prior carotid endarterectomy. J Vasc Surg. 2005; 41(6): 956-961, doi: 10.1016/j.jvs.2005.02.038, indexed in Pubmed: 15944593.

6. Choi SS, Lim YJ, Bahk JH, et al. Coronary artery spasm induced by carotid sinus stimulation during neck surgery. $\mathrm{Br}$ J Anaesth. 2003; 90(3): 391-394, doi: 10.1093/bja/aeg051.

7. Dehn T, Angell-James JE. Long-term effect of carotid endarterectomy on carotid sinus baroreceptor function and blood pressure control. Br J Surg. 1987; 74(11): 997-1000, doi: 10.1002/bjs.1800741113.

8. Eastcott HH, Pickering GW, Rob CG. Reconstruction of internal carotid artery in a patient with intermittent at- 
tacks of hemiplegia. Lancet. 1954; 267(6846): 994-996, doi: 10.1016/S0140-6736(54)90544-9, indexed in Pubmed: 13213095.

9. Hirschl M, Kundi M, Hirschl $M$, et al. Blood pressure responses after carotid surgery: Relationship to postoperative baroreceptor sensitivity. Am J Med. 1993; 94(5): 463-468, doi: 10.1016/0002-9343(93)90079-5.

10. Kurkcuoglu A, Aytekin C, Oktem H, et al. Morphological variation of carotid artery bifurcation level in digital angiography. Folia Morphol. 2015; 74(2): 206-211, doi: 10.5603/ FM.2015.0032, indexed in Pubmed: 26050808.

11. Leisch F, Kerschner K, Hofmann R, et al. Carotid sinus reactions during carotid artery stenting: predictors, incidence, and influence on clinical outcome. Catheter Cardiovasc Interv. 2003; 58(4): 516-523, doi: 10.1002/ccd.10483, indexed in Pubmed: 12652504.

12. Lucev N, Bobinac D, Maric I, et al. Variations of the great arteries in the carotid triangle. Otolaryngol Head Neck Surg. 2000; 122(4): 590-591, doi: 10.1067/mhn.2000.97982, indexed in Pubmed: 10740186.

13. Marrocco-Trischitta MM, Cremona G, Lucini D, et al. Peripheral baroreflex and chemoreflex function after eversion carotid endarterectomy. J Vasc Surg. 2013; 58(1): 136-44.e1, doi: 10.1016/j.jvs.2012.11.130, indexed in Pubmed: 23632295.

14. Michalinos A, Chatzimarkos M, Arkadopoulos N, et al. Anatomical considerations on surgical anatomy of the carotid bifurcation. Anat Res Int. 2016; 2016: 6907472, doi: 10.1155/2016/6907472, indexed in Pubmed: 27047690.

15. Nishizaki M, Yamawake N, Arita M. Coronary artery spasm induced by carotid sinus massage. Heart. 2000; 84(1): E2, doi: 10.1136/heart.84.1.e2, indexed in Pubmed: 10862603.

16. Schulz U, Rothwell PM. Major variation in carotid bifurcation anatomy: a possible risk factor for plaque development? Stroke. 2001; 32(11): 2522-2529, doi: 10.1161/ hs1101.097391.
17. Sigaudo-Roussel D, Evans DH, Naylor AR, et al. Deterioration in carotid baroreflex during carotid endarterectomy. J Vasc Surg. 2002; 36(4): 793-798, doi: 10.1067/ mva.2002.126564, indexed in Pubmed: 12368741.

18. Smith $D$, Larsen JL. On the symmetry and asymmetry of the bifurcation of the common carotid artery: a study of bilateral carotid angiograms in 100 adults. Neuroradiology. 1979; 17(5): 245-247, doi: 10.1007/BF00337533, indexed in Pubmed: 481739.

19. Tang TY, Walsh SR, Gillard JH, et al. Carotid sinus nerve blockade to reduce blood pressure instability following carotid endarterectomy: a systematic review and meta-analysis. Eur J Vasc Endovasc Surg. 2007; 34(3): 304-311, doi: 10.1016/j. ejvs.2007.02.024, indexed in Pubmed: 17509910.

20. Toorop RJ, Ousrout R, Scheltinga MRM, et al. Carotid baroreceptors are mainly localized in the medial portions of the proximal internal carotid artery. Ann Anat. 2013; 195(3): 248-252, doi: 10.1016/j.anat.2012.12.001, indexed in Pubmed: 23452666.

21. Toorop RJ, Scheltinga MR, Moll FL, et al. Anatomy of the carotid sinus nerve and surgical implications in carotid sinus syndrome. J Vasc Surg. 2009; 50(1): 177-182, doi: 10.1016/j.jvs.2009.03.029, indexed in Pubmed: 19563966.

22. Toorop RJ, Visser RF, Moll FL, et al. Long term safety and efficacy of internal carotid artery adventitial stripping in carotid sinus syndrome. Curr Hypertens Rev. 2014; 10(1): 58-64, doi: 10.2174/157340211001141111161240, indexed in Pubmed: 25392145.

23. Yip HK, Sung $\mathrm{PH}, \mathrm{Wu} \mathrm{CJ}$, et al. Carotid stenting and endarterectomy. Int J Cardiol. 2016; 214: 166-174, doi: 10.1016/j.ijcard.2016.03.172, indexed in Pubmed: 27061654.

24. Zhang C, Xie S, Li S, et al. Flow patterns and wall shear stress distribution in human internal carotid arteries: the geometric effect on the risk for stenoses. J Biomech. 2012; 45(1): 83-89, doi: 10.1016/j.jbiomech.2011.10.001, indexed in Pubmed: 22079384. 